\title{
Indicateurs du contenu " matières premières et énergie " des déchets de démolition
}

\author{
Nicolas Roussat, Jacques Méhu \\ LGCIE, INSA-Lyon \\ 20, avenue Albert-Einstein, 6962 I Villeurbanne Cedex \\ Pour tout contact : 0472436361 ou nicolas.roussat@insa-lyon.fr
}

\begin{abstract}
Cette étude a pour objectif de mettre en place des indicateurs afin d'évaluer le contenu « matières premières et énergie » des déchets issus de la démolition de bâtiments. Ce contenu est caractérisé d'une part par la totalité des matières premières et de l'énergie nécessaires à la fabrication d'un bâtiment (désigné respectivement par intensité matérielle et énergie grise), et d'autre part par le caractère « épuisable » des matériaux contenus dans les déchets de démolition. Ces caractéristiques « contenu matières premières et énergie » sont des indicateurs d'impacts environnementaux globaux tels que l'épuisement des ressources naturelles abiotiques et la consommation d'énergie non renouvelable. Ils permettent, grâce à un bilan matière et énergie, de mesurer la performance environnementale globale des filières de traitement de déchets, performance qui sera ensuite utilisée comme critère de décision dans le cadre d'une recherche sur l'optimisation des stratégies de gestion des déchets de démolition.
\end{abstract}

Mots clés : énergie grise, intensité matérielle, matières premières non renouvelables, déchets du bâtiment, produits de construction

\section{Introduction}

Les produits de construction, comme tous produits manufacturés, ont une valeur qui est liée à la quantité et à la rareté des matières nécessaires à leur fabrication, ainsi qu'à l'énergie utilisée lors de cette fabrication. La valeur d'un produit est donc dépendante de ce contenu « matières premières et énergie » lié à sa fabrication.

Durant la vie d'un bâtiment, chacun de ces produits a un usage, une valeur d'utilité qui justifie l'investissement environnemental initial en termes de matière première et d'énergie. Mais lorsqu'un bâtiment est en fin de vie, lorsqu'il devient obsolète ou hors d'usage, ces produits deviennent des déchets, des rebuts, et perdent leur usage, leur valeur d'utilité.

Pourtant, ces produits devenus déchets ont encore une valeur économique mais aussi environnementale car :

- l'extraction de matières premières et la consommation d'énergie et d'eau liées à la fabrication de ces produits peut représenter un coût environnemental important ;

- ces produits peuvent contenir des matériaux non renouvelables, dont il est important d'optimiser l'utilisation compte tenu de leur rareté et de leur caractère épuisable. En s'inspirant de la doctrine de l'écologie industrielle, un bâtiment démoli ne doit pas être perçu comme un gisement de déchets mais comme un stock potentiel de matières premières, utilisable à travers le recyclage et la valorisation de ces déchets.

Afin de caractériser ce contenu « matières premières et énergie », avec l'objectif de cibler les déchets de démolition ayant un coût environnemental important (et qu'il faut donc valoriser ou recycler en priorité) et d'évaluer les performances environnementales des différentes filières de traitement, plusieurs indicateurs concernant l'énergie et les matières premières ont été mis en place. Ces indicateurs sont présentés dans les paragraphes suivants.

\section{Contenu " énergie " : l'énergie grise des déchets de démolition}

Tout bien matériel créé par l'homme nécessite un apport d'énergie. Notre activité économique a donc une relation directe avec l'énergie. De ce fait, l'économie, comme tout système utilisant de l'énergie, ne peut échapper au second principe de la thermodynamique. Rappelons que le second principe de la thermodynamique, énoncé par Carnot, introduit la notion d'entropie. L'entropie représente la dégradation de l'énergie, qui intervient lors des processus irréversibles (par exemple, l'énergie utilisée pour chauffer un fluide ne peut être utilisée qu'en partie pour obtenir de l'énergie mécanique, la partie « dégradée » correspondant à un accroissement de l'entropie).

En fait, on peut considérer que l'économie ne cesse de créer de l'entropie, car quel que soit le bien matériel produit, il est impossible de récupérer totalement l'énergie qui a été nécessaire à sa fabrication. En effet, cette énergie se disperse et se dégrade forcément par l'usure et la destruction du bien dans un processus irréversible. 
Comme le précise Passet [I], « les substances relativement organisées (minerais, combustibles...), prélevées en amont du système économique..., se trouvent en définitive toujours restituées en aval sous des formes (fumées, cendres, déchets...) déstructurées ».

Cette déstructuration de la matière, et donc cette dégradation de l'énergie, a un impact. Car bien que notre biosphère soit alimentée par une source d'énergie illimitée à l'échelle humaine (celle qui provient du soleil), les stocks d'énergie utilisés aujourd'hui (surtout les combustibles fossiles) sont limités. Donc toute l'énergie utilisée pour la fabrication des produits de construction est soumise à la dégradation entropique. Les produits de construction n'ont donc pas seulement une valeur économique, ils ont aussi une valeur énergétique. II en va de même pour les déchets de construction, qui constituent une perte nette du point de vue énergétique, et augmentent donc l'entropie du système. II est essentiel, dans une optique de développement durable, de minimiser cette génération d'entropie. Une possibilité de la diminuer, outre l'optimisation des moyens de production, est d'économiser de l'énergie en utilisant les déchets pour la fabrication de nouveaux produits, permettant ainsi d'économiser la consommation énergétique due à l'extraction ou au raffinage des minerais.

Le contenu énergétique des déchets de démolition est alors mesuré avec l'énergie grise (ou embodied energy). Ce contenu n'a aucun rapport avec l'éventuel pouvoir calorifique du déchet ; il représente l'énergie utilisée pour la fabrication du produit, de l'extraction des matières premières au produit fini. II représente l'investissement énergétique lié à la fabrication du produit.

\section{Contenu « matières premières $\|$ des déchets de démolition}

Après avoir défini le contenu « énergie » des déchets de démolition avec le concept d'énergie grise, on définit ici les indicateurs permettant de mesurer le contenu « matières premières » des déchets de démolition.

Le premier indicateur relatif au contenu « matières premières » de déchets concerne l'extraction des matières premières nécessaires à la fabrication des matériaux de construction. Celui-ci sera évalué à l'aide de l'indicateur nommé intensité matérielle. Ce premier indicateur nous renseigne sur la totalité des matières premières qu'il a fallu extraire pour construire un bâtiment.

Le deuxième indicateur concerne la valeur (ou la rareté) du contenu « matières premières » des déchets en tant que tels. On ne s'intéresse plus ici à la fabrication du produit mais seulement à son contenu. Le bâtiment démoli est ici perçu comme un stock de matières premières, un gisement potentiellement exploitable dont on cherche à connaître la nature et la richesse des ressources à exploiter. Ce facteur se traduira par un indicateur des ressources naturelles potentielles, relatif à chaque constituant présent dans les déchets de démolition. Cet indicateur mesure le caractère rare et non renouvelable des matériaux composant le bâtiment, et permet donc de hiérarchiser les déchets à exploiter prioritairement, compte tenu de leur caractère épuisable.

\section{MIPS : indicateur de l'intensité matérielle par unité de service}

On définit l'intensité matérielle par la masse totale de matières premières prélevées dans l'environnement et nécessaires à la fabrication d'un produit.

Ce concept a été développé en Allemagne par le Wuppertal Institut [3], qui a proposé un indicateur appelé MIPS (material input per unit of service, ou intensité matérielle par unité de service). II s'agit d'un outil très proche de l'ACV (analyse du cycle de vie) puisqu'il est basé sur l'ensemble des flux de matières entrant en jeu lors de la conception d'un produit. Mais contrairement à l'ACV où l'on considère l'ensemble des impacts liés au cycle de vie du produit, on ne considère ici que le flux de matières entrant dans la conception du produit.

En combinant l'énergie grise et l'intensité matérielle des produits constituant un bâtiment, on couple un flux énergétique et un flux de matières, qui donnent à eux deux une bonne image des impacts environnementaux globaux (effet de serre, épuisement des ressources naturelles, altération des milieux physiques, amenuisement de la biodiversité...). En effet, l'intensité matérielle ne concerne pas seulement l'épuisement des ressources naturelles. L'essentiel de la production minière actuelle est à ciel ouvert, ce qui implique, avant toute exploitation, la destruction de l'écosystème où se situe le minerai. Donc plus un produit a une intensité matérielle élevée, plus il participe à l'altération du milieu naturel.

Le MIPS représente la quantité de matière utilisée pour une unité de service donnée (cette unité de service peut directement être reliée à l'unité fonctionnelle de l'ACV). L'unité de service peut être très variable. Par exemple, elle peut être de 3000 cycles de lavage pour une machine à laver, ou 400000 $\mathrm{km}$ pour une automobile, etc. En fait, on ramène la quantité de matière utilisée au service fourni, ce qui permet de définir les produits les plus respectueux de l'environnement pour un service donné.

Dans notre cas, on ne cherche pas à définir les matériaux de construction les plus respectueux de l'environnement, mais à définir quels sont les déchets issus de la démolition qui représentent des enjeux importants, compte tenu de leurs coûts environnementaux, et qui doivent donc faire l'objet d'efforts importants de valorisation. On ne prendra donc pas en compte ici la notion de service car par définition, un déchet ne fournit aucun service. On ne considérera que la notion d'intensité matérielle, sans la ramener au service fourni.

Le Wuppertal Institut divise l'intensité matérielle en cinq catégories différentes qui sont:

- les matières premières abiotiques, qui représentent les matières premières non renouvelables comme les minéraux (gravier, sable, minerai...), les énergies fossiles, mais aussi tous les résultats de forage et d'extraction non utilisés mais 
néanmoins consommés indirectement par la production du produit ( I tonne d'acier ne nécessite pas uniquement I tonne de minerai !) ;

- les matières premières biotiques, qui sont renouvelables et représentent la biomasse, cultivée ou non ;

- les mouvements de terres provoqués par l'agriculture,

l'exploitation forestière, la construction... ;

— l'eau consommée pour la production du produit ;

- l'air utilisé, que se soit pour la combustion ou pour une transformation chimique.

L'intensité matérielle calcule donc le flux de matières qui passe de l'écosphère à la technosphère (du milieu naturel au milieu anthropique), c'est-à-dire les matières premières extraites du milieu naturel pour la fabrication d'un produit.

\section{L'intensité matérielle, indicateur de la dématérialisation des produits?}

La dématérialisation de l'économie, concept fondamental de l'écologie industrielle [4], consiste à minimiser les flux totaux de matières prélevées dans l'environnement pour la fabrication d'un produit, tout en assurant un service et un usage équivalent. De manière générale, la dématérialisation de l'économie passe par l'optimisation de l'utilité du produit, autrement dit par la commercialisation de l'usage plutôt que de l'objet. L'intensité matérielle, telle qu'elle a été définie, représente le flux total de matière pour l'élaboration d'un produit. La dématérialisation d'un produit se traduit donc par une diminution de son intensité matérielle.

Une des clés de la dématérialisation de l'économie, c'est-àdire de la réduction des flux de matières entre le milieu nature et anthropique, passe par la gestion des déchets. Le recyclage et la valorisation matière des déchets constituent clairement une voie de dématérialisation de l'économie. Par exemple, on peut comparer l'intensité matérielle due à la production de l'aluminium et de l'acier à partir de minerai, et l'intensité de ces mêmes métaux produits avec des matières recyclées. En termes de matériaux abiotiques, l'économie réalisée grâce au recyclage par rapport à une production normale est de $84 \%$ dans le cas de l'acier et de $98 \%$ dans le cas de l'aluminium ${ }^{[5]}$.

Et si cela est particulièrement vrai pour les métaux, compte tenu de la proportion importante de minerai à extraire pour leur production, cela reste a fortiori vrai pour les autres produits issus de la démolition.

Le calcul de l'intensité matérielle, par produit, d'un bâtiment, nous permettra donc aussi de déterminer les déchets pour lesquels le recyclage permettrait une dématérialisation importante (a priori, plus l'intensité matérielle est importante, plus le recyclage permettra une dématérialisation significative).

\section{Indicateur des ressources naturelles potentielles}

Le système anthropique, comme tous les systèmes physiques, répond aux lois de la physique, et notamment à la loi de la conservation de la masse. Donc tout ce qui entre dans ce système, finit par sortir du système ou s'accumule dans celuici. Mais si la masse des matériaux prélevés dans l'environnement ne se perd pas, ces matériaux peuvent perdre leur valeur selon leur usage et donc leur valeur économique. Par exemple, des briques utilisées pour la construction d'un immeuble entrent dans le système en ayant une valeur commerciale, puis elles restent dans le système pendant la durée de vie du bâtiment. Lorsque le bâtiment est démoli, les déchets liés aux briques, qui contiennent pourtant les mêmes matériaux que les briques initiales, non plus aucune valeur commerciale et, pire, on peut dire qu'ils ont une valeur négative, leur traitement ou leur stockage ayant un coût. II est évident que les déchets de briques ne peuvent pas avoir la même valeur que des briques neuves. Il y a un phénomène de dégradation entropique : on passe d'un état de la matière organisé (les briques) à un état dégradé (les briques usées, cassées ou souillées à l'état de déchets). Dans la plupart des cas, la perte de valeur est totale. Pourtant, ces déchets contiennent de la matière qui peut encore avoir une utilisation ou un usage, une matière potentiellement exploitable. Les déchets devraient donc garder une certaine valeur, être considérés au moins comme du minerai. « Les entreprises et les pouvoirs publics devraient donc commencer à considérer d'un autre œil les montagnes de déchets qui s'accumulent dans les décharges: non plus comme des immondices à faire disparaître, mais comme de véritables gisements de matières premières qu'il sera possible d'exploiter un jour. Dans l'optique de l'écologie industrielle, les décharges ne sont rien d'autre que des mines artificielles ! $\gg{ }^{[4]}$

Les déchets de la démolition représentent donc un stock de matières, équivalent à une mine de matériaux inexploitée à ce jour, car perçue comme sans valeur. L'évaluation de la valeur de ce gisement potentiel s'appuie sur le caractère épuisable et non renouvelable des matériaux contenus dans le bâtiment démoli. Plus les matériaux sont rares, et donc épuisables rapidement, plus leur exploitation à partir du gisement des déchets de démolition présentent de l'intérêt

Le caractère épuisable et non renouvelable des matériaux contenus dans les déchets de démolition a été évalué avec l'aide de la norme NF P OI-0 I $0{ }^{[2]}$ concernant « la déclaration environnementale et sanitaire des produits de construction », et plus particulièrement les paragraphes liés à l'épuisement des ressources naturelles.

Contrairement à la norme, qui considère l'ensemble du cycle de vie des produits de construction, nous ne considérons ici que les matériaux contenus dans les déchets, sans nous intéresser aux modes de fabrication des produits. Nous nous intéressons seulement à la valeur du gisement potentiel de matières premières que représentent les déchets de démolition. On ne cherche pas ici à connaître les impacts évités par le recyclage ou la valorisation des produits (l'énergie grise et l'intensité matérielle en sont des indicateurs), mais à évaluer la valeur « minière » que représente les déchets. Pour évaluer cette valeur, on considère les différents éléments constituants un produit de construction. Ces différents éléments sont pondérés par les coefficients de conversion de la norme NF P OI-0I0. Ces coefficients sont issus de la méthode introduisant l'indicateur ADP (abiotic depletion potential) développée par l'Institute of Environmental Sciences (CML) de Lendein University ${ }^{[6]}$ Cette méthode est basée sur le principe des flux équivalents. Le principe consiste à convertir un flux d'une substance d'origine 
en un flux d'une substance de référence propre à la catégorie d'impact que l'on souhaite évaluer. L'ADP est évalué en fonction de l'état des réserves de la ressource et de son taux d'extraction. Ce facteur est basé sur l'état des réserves « ultimes » des éléments, c'est-à-dire sur la concentration des éléments, dont on cherche à mesurer l'épuisement dans la couche terrestre. L'indicateur est calculé pour chaque élément et normalisé par rapport à un élément de référence qui est l'antimoine. Au final, pour chaque produit de construction, on multiplie la masse des éléments qui le composent par leurs indicateurs ADP. On obtient alors une masse d'antimoine équivalente. Plus cette masse équivalente est élevée, plus le produit est considéré comme rare et épuisable, et plus l'exploitation des déchets en tant que matières premières secondaires est stratégique.

\section{Bilan matière et énergie}

L'objectif de ce bilan est de quantifier l'impact environnemental global d'un scénario de traitement de déchets de démolition d'un bâtiment, afin de le comparer à d'autres scénarios possibles de gestion de ces déchets de démolition. Ce bilan se base sur les indicateurs définis auparavant pour quantifier le contenu « matières premières et énergie » des déchets de démolition. La figure I et le tableau I présentent les différents flux considérés pour ce bilan.

Chaque déchet est caractérisé initialement par son énergie grise, son intensité matérielle et son indicateur de ressources naturelles potentielles.

\begin{tabular}{|l|l|}
\hline Egi & Énergie grise du déchet $\mathrm{i}$ \\
\hline $\mathrm{Ei}$ & Énergie récupérée lors de la valorisation du déchet $\mathrm{i}$ \\
\hline Egji & Énergie grise du produit $\mathrm{j}$ fabriqué de manière traditionnelle \\
\hline Evi & Énergie nécessaire à la valorisation matière du déchet $\mathrm{i}$ \\
\hline Eri & Énergie nécessaire au recyclage du déchet $\mathrm{i}$ \\
\hline IMi & Intensité matérielle du déchet $\mathrm{i}$ (en tonnes) \\
\hline IMepi & $\begin{array}{l}\text { Intensité matérielle en équivalent pétrole de l'énergie Ei récupérée (économie de } \\
\text { pétrole due à la valorisation énergétique) }\end{array}$ \\
\hline IMji & Intensité matérielle du produit j fabriqué de manière traditionnelle \\
\hline IMvi & Intensité matérielle nécessaire à la valorisation matière du déchet $\mathrm{i}$ \\
\hline IMri & Intensité matérielle nécessaire au recyclage du déchet $\mathrm{i}$ \\
\hline Iri & Indicateur de ressources potentielles contenues dans le déchet \\
\hline Ircombi & Indicateur de ressources potentielles combustibles dans le déchet $\mathrm{i}$ \\
\hline Irji & Indicateur de ressources potentielles utilisées pour la fabrication du produit $\mathrm{j}$ \\
\hline Irri & Indicateur de ressources potentielles utilisées pour le recyclage \\
\hline
\end{tabular}

Tableau I : notations utilisées pour le bilan

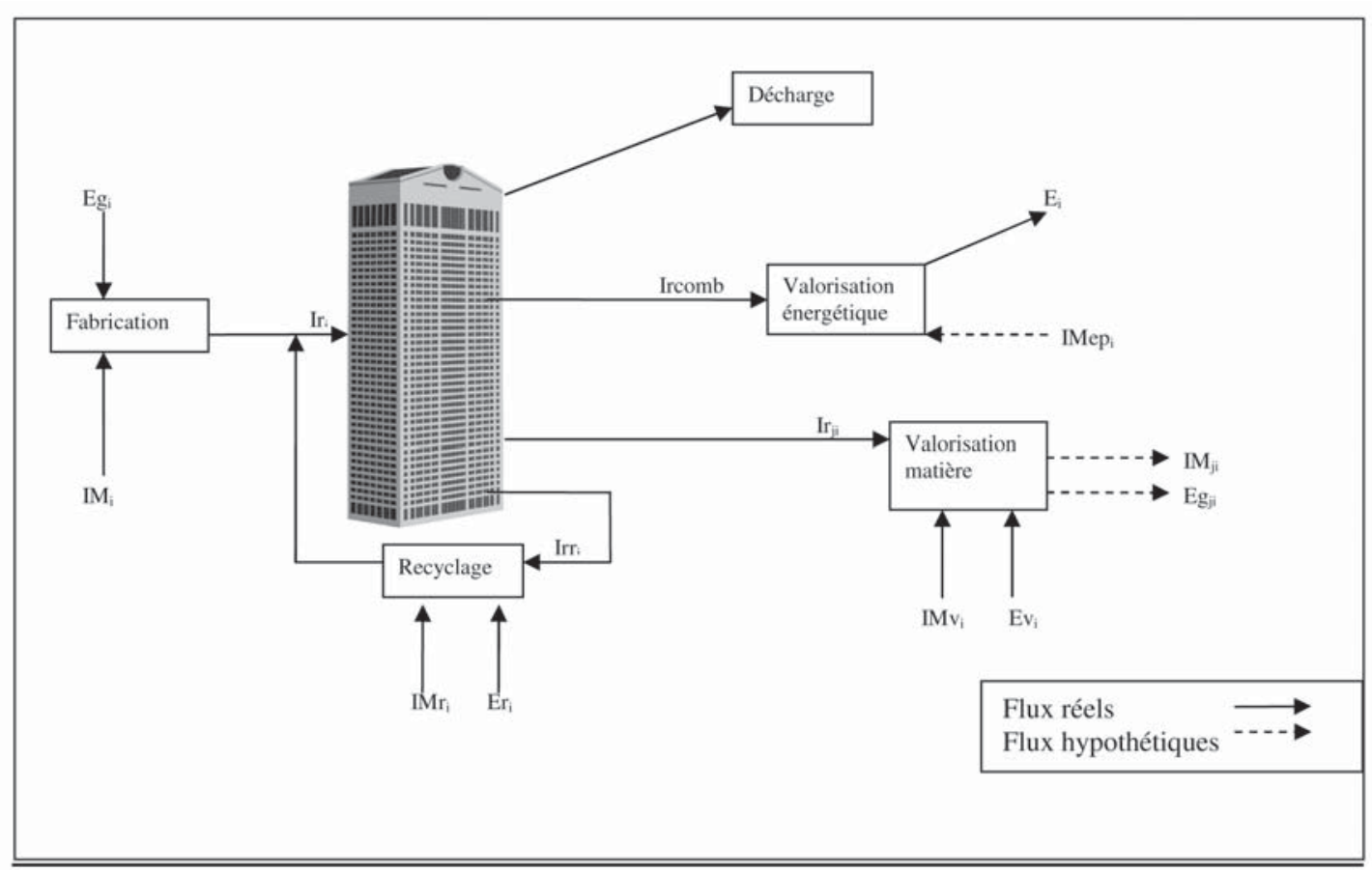

Figure I : bilans masse et énergie 
Les déchets peuvent être dirigés selon quatre voies:

— soit ils partent en décharge ;

— soit ils peuvent être valorisés énergétiquement, et fournir une énergie $E$. Dans ce cas, on peut estimer que l'on a économisé un flux hypothétique d'énergie fossile qui aurait été nécessaire à la production de la même énergie. On considère ici que le déchet valorisé énergétiquement représente un substitut au pétrole, et donc que l'on économise le pétrole qui aurait été nécessaire à la production de l'énergie $E$;

— soit ils sont utilisés pour une valorisation matière qui conduit à la fabrication d'un nouveau produit j. Dans ce cas, on a économisé les flux matières et énergie qui auraient été nécessaires à la fabrication du produit j si celui-ci avait été fabriqué de manière traditionnelle, à partir de matières naturelles. On prend aussi en compte l'énergie et l'intensité matérielle nécessaire à cette valorisation ;

— soit les déchets sont recyclés. Ils reprennent donc leur valeur initiale Mais le recyclage nécessite un apport d'énergie et de matières premières qui font que le bilan masse et énergie n'est pas nul. (1)

Le bilan permet de connaître les performances environnementales globales des filières de traitement, vis-à-vis de l'investissement environnemental initial en termes d'utilisation de matières premières, d'épuisement des ressources et de consommation énergétique. Ces performances environnementales sont mesurées à l'aide des trois indicateurs présentés ci-dessous.

\section{Énergie perdue}

Cet indicateur considère l'énergie totale consommée par le système anthropique, de l'investissement énergétique initial pour la fabrication du produit à la gestion finale du déchet. Cette énergie « perdue » est calculée par un bilan énergétique sur les procédés de traitement et de valorisation.

Pour le recyclage, la seule énergie perdue correspond à l'énergie nécessaire au recyclage. En effet, l'énergie grise initiale du déchet se retrouve dans le nouveau produit, elle n'est donc pas perdue. Pour la valorisation matière, l'énergie perdue correspond à la somme de l'énergie grise initiale du déchet i et de l'énergie nécessaire à la valorisation, moins l'énergie qui aurait été nécessaire à la fabrication du produit j de manière traditionnelle, c'est-à-dire à partir de matières premières prélevées dans l'environnement.

Pour la valorisation énergétique, on compare l'énergie grise initiale à la production d'énergie.

Pour la mise en décharge, on considère que l'on perd la totalité de l'énergie grise initiale.

Pour le recyclage : $\Delta \mathrm{E}=\mathrm{E} \mathrm{r}_{1}$

Pour la valorisation matière $: \Delta \mathrm{E}=\mathrm{Eg}+\mathrm{EV}_{\mathrm{i}}-\mathrm{Eg}_{\mathrm{i}}$

Pour la valorisation énergétique $: \Delta \mathrm{E}=\mathrm{Eg}$ - $\mathrm{Ei}$

Pour la mise en décharge $: \Delta \mathrm{E}=\mathrm{Eg}$

\section{Intensité matérielle perdue}

Cet indicateur permet de mesurer l'intensité matérielle qui a été perdue selon la filière choisie pour le déchet. Cet indicateur, comme le précédent, est basé sur la comparaison entre les flux entrants dans les filières et les flux sortants (que ceux-ci soient hypothétiques ou réels).

Pour le recyclage, l'intensité matérielle perdue correspond à l'intensité matérielle nécessaire au recyclage. Comme pour l'énergie, l'intensité matérielle initiale du déchet se retrouve en totalité dans le produit recyclé.

Pour la valorisation matière, le flux entrant correspond à l'intensité matérielle initiale du déchet i additionnée de l'intensité matérielle nécessaire à la valorisation. Le flux sortant, hypothétique, correspond à l'intensité matérielle qui aurait été nécessaire à la fabrication du produit j si celui-ci avait été fabriqué de manière traditionnelle.

Pour la valorisation énergétique, l'intensité matérielle perdue correspond à l'intensité matérielle initiale, diminuée de l'intensité matérielle de la quantité de pétrole qui aurait fourni la même énergie.

Pour la mise en décharge, la totalité de l'intensité matérielle initiale est perdue.

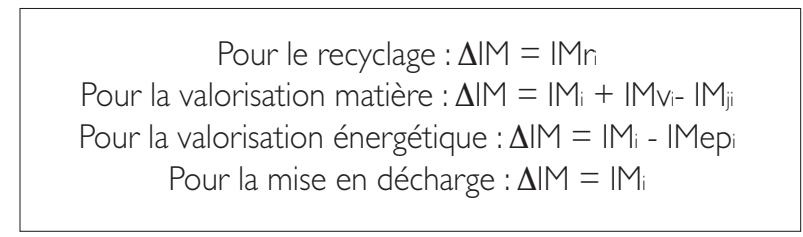

\section{Taux d'utilisation des ressources naturelles potentielles}

Cet indicateur représente le taux de matières non renouvelables contenues dans le déchet qui a été valorisé. C'est en fait un taux de recyclage pondéré par le facteur d'épuisement de chaque ressource.

Pour le recyclage, ce taux correspond à la quantité de ressources présentes dans le déchet i qui est recyclée, divisée par la quantité totale de ressources présentes dans le déchet i (la différence entre les deux correspond aux pertes relatives aux procédés de recyclage, aux pertes durant la déconstruction, etc.) Pour la valorisation matière, le taux de matériaux valorisés correspond aux ressources utilisées pour la fabrication du nouveau produit j par rapport aux matériaux contenus dans le déchet i.

Pour la valorisation énergétique, seuls les matériaux combustibles sont valorisés. Le taux d'utilisation de ressources correspond donc aux matériaux combustibles présents dans le déchet (qui sont les matériaux utiles à la combustion, donc les seuls valorisés) sur les ressources présentes initialement dans le déchet i. Pour la mise en décharge, aucune ressource présente initialement dans le déchet n'est utilisée, le taux d'utilisation est donc nul.

Pour le recyclage $: \operatorname{Trp}=\mid \mathrm{Ir}_{1} / \mathrm{I} \mathrm{r}_{\mathrm{I}}$

Pour la valorisation matière $: \operatorname{Trp}=\mid \mathrm{r}_{\mathrm{ji}} / \mathrm{Ir}$

Pour la valorisation énergétique $: \operatorname{Trp}=1$ rcombi / Ir

Pour la mise en décharge :Trp $=0 \%$ 


\section{Perspectives}

Ces trois indicateurs (énergie grise, intensité matérielle et ressources naturelles potentielles) permettent de mesurer les impacts environnementaux globaux liés à la gestion des déchets de démolition à l'aide d'un bilan matière et énergie. La connaissance de ces impacts permet de les intégrer comme critère de décision dans une analyse multicritère ayant pour objectif d'optimiser la gestion des déchets de démolition visà-vis du développement durable. Bien sûr, ces indicateurs ne sont nullement suffisants pour considérer l'ensemble de la problématique du développement durable et doivent être complétés par des indicateurs concernant les impacts environnementaux locaux (pollution du sol et de l'eau, impacts sanitaires...) et les aspects socio-économiques liés à la gestion des déchets.

Ces indicateurs sont actuellement utilisés dans le cadre d'une étude réalisée en partenariat avec le Grand Lyon afin d'évaluer la gestion des déchets de démolition générés sur le territoire du Grand Lyon. Cette étude, basée sur le suivi de plusieurs chantiers de démolition, doit permettre de définir le ou les modes de gestion des déchets de démolition les plus performants vis-à-vis des critères du développement durable.

\section{Remerciements}

Ce travail est réalisé dans le cadre d'une thèse subventionnée par la région Rhône-Alpes, en partenariat avec le Grand Lyon.

\begin{abstract}
Note :
I. Bien que la plupart du temps (et notamment dans les publications de l'Ademe et du ministère de l'Ecologie), aucune distinction ne soit faite entre le recyclage et la valorisation matière, nous retenons, dans cet article, la distinction suivante :

Le recyclage est une opération qui ramène le déchet sous la forme du produit initial, alors que la valorisation matière utilise le déchet comme produit de substitution à un autre produit.

Par exemple, les déchets de verre sont généralement recyclés. Ils servent à produire du calcin qui sert à nouveau à produire du verre (donc le même produit). Par contre, l'utilisation de mâchefers d'usine d'incinération en tant que sous-couche routière constitue une valorisation matière car les mâchefers se substituent à des granulats naturels. Dans ce dernier cas, nous ne parlons pas de recyclage puisque les déchets ont une utilisation différente des produits initiaux. Ainsi, selon notre définition, le recyclage est une valorisation matière, mais toutes les valorisations matières ne correspondent pas forcément à une opération de recyclage.
\end{abstract}

\section{Bibliographie}

[I] PASSET René. L'économique et le vivant. 2e éd., Paris : Ed. Economica, 1996, 29I p.

[2] AFNOR. Qualité environnementale des produits de construction. Déclaration environnementale et sanitaire des produits de construction. NF P OI-0 I0. Paris : AFNOR, 2004, 47 p.

[3] RITTHOFF M, ROHN H, LIEDTKE. Calculating MIPS - Resource productivity of products and services. [en ligne] Wuppertal :Wuppertal Institute for Climate, Environment and Energy, 2003. Disponible sur : www.mips-online.info (consulté le 18/06/05)

[4] ERKMAN Suren.Vers une écologie industrielle. 2e édition, enrichie et mise à jour Paris : Edition Charles Leopold Mayer, 2004, 25I p.

[5] Wuppertal Institute for Climate, Environment and Energy. Tables containing material intensity factors of material and energy source. [en ligne] Wuppertal :Wuppertal Institute for Climate, Environment and Energy, 2003. Disponible sur : www.mips-online.info

[6] VAN OERS L, de KONING A, GUINEE JB, HUPPES G. Abiotic resource depletion in LCA - Improving characterisation factors for abiotic resource depletion as recommended in the new Dutch LCA Handbook. [en ligne] Leinden (Pays-Bas): the Road and Hydraulic Engineering Institute of the Dutch Ministry of Transport, Public Works and Water Management, 2002. Disponible sur : www.leidenuniv.nl/cml/ssp/projects/lca2/report_abiotic_depletion_web. pdf

[7] ATKINSON C, HOBBS J,WEST J, EDWARDS S. Life cycle embodied energy and carbon dioxide emissions in buildings. Industry and environment, 1996, Vol. 19, n² 2, pp. 29-32.

[8] CRAIGHILL A, POWELL J.C.A lifecycle assessment and evaluation of construction and demolition waste, CSERGE working paper WM 99-03 [en ligne] London : CSERGE (Centre for Social and Economic Research on the Global Environment), 1999. Disponible sur : < http://www.uea.ac.uk/env/cserge/pub/wp/wm/wm_1999_03.pdf>.

[9] DE BEIR Jean. Recyclage et dématérialisation de l'économie : « La croissance économique devient-elle immatérielle? Réflexion sur une voie possible de la durabilité ». [en ligne] Disponible sur : www.erasme.ecp.fr/session3/De\%Beir.pdf (consulté le 20/06/05)

[10] HAAKE Julia. Dématérialisation: Mesure par bilan matière et MIPS, In : Technique de l'ingénieur environnement- Outils de gestion Paris :Techniques de l'ingénieur, 2000, pp. G5910-I - G5910-8.

[I I] PIETERS G. The construction industry and the environment in Europe. Industry and environment, 1996, Vol. 19, n² 2, pp. 9- 12.

[12] SCHEUER C, KEOLEIAN G.A, REPPE P. Life cycle energy and environmental performance of a new university building : modelling challenges and design implications. Energy and building : Elsevier, 2003, Vol. 35, n II, pp. 1049-1064.

[ I 3]VENKATARAMA REDDY B.V,JAGADISH K.S. Embodied energy of common and alternative building materials and technologies. Energy and building : Elsevier, 2003,Vol. 35, n², pp. $129-137$.

[14] YOHANIS Y.G, NORTON B. Life-cycle and embodied energy for a generic single storey office building in the UK. Energy - Oxford. Pergamon, 2002, Vol 27, n I, pp. 77-92. 